\title{
A novel and economical explanation for SM fermion masses and mixings
}

\author{
A. E. Cárcamo Hernández ${ }^{a}$ \\ Universidad Técnica Federico Santa María and Centro Científico-Tecnológico de Valparaíso, Casilla 110-V, Valparaíso, Chile
}

Received: 16 June 2016 / Accepted: 2 September 2016 / Published online: 15 September 2016

(c) The Author(s) 2016. This article is published with open access at Springerlink.com

\begin{abstract}
I propose the first multiscalar singlet extension of the standard model (SM), which generates tree level top quark and exotic fermion masses as well as one and three loop level masses for charged fermions lighter than the top quark and for light active neutrinos, respectively, without invoking electrically charged scalar fields. That model, which is based on the $S_{3} \times Z_{8}$ discrete symmetry, successfully explains the observed SM fermion mass and mixing pattern. The charged exotic fermions induce one loop level masses for charged fermions lighter than the top quark. The $Z_{8}$ charged scalar singlet $\chi$ generates the observed charged fermion mass and quark mixing pattern.
\end{abstract}

\section{Introduction}

Despite its great consistency with the experimental data, the standard model (SM) is unable to explain several issues such as, for example, the number of fermion generations, the observed pattern of fermion masses and mixings, etc. In this letter I propose the first multiscalar singlet extension of the SM, that generates tree level top quark and exotic fermion masses as well as one and three loop level masses for charged fermions lighter than the top quark and for light active neutrinos, respectively, without invoking electrically charged scalar fields. That multiscalar singlet extension is consistent with the SM fermion mass and mixing pattern.

\section{The model}

The model has the SM gauge symmetry, which is supplemented by the $S_{3} \times Z_{8}$ discrete group. It is noteworthy that among the discrete symmetries, I introduced the symmetry group $S_{3}$ since it is the smallest non-Abelian group that has been considerably studied in the literature. The $S_{3}$ symmetry

a e-mail: antonio.carcamo@usm.cl is assumed to be preserved whereas the $Z_{8}$ discrete group is broken at the scale $v_{\chi}$. The breaking of the $Z_{8}$ symmetry gives rise to the observed charged fermion mass and quark mixing pattern. The scalar sector of the SM is extended by introducing three EW scalar singlets, i.e., $\eta_{1}, \eta_{2}$, and $\chi$, assumed to be charged under the $Z_{8}$ symmetry. Out of these three SM scalar singlets, two scalar fields $\left(\eta_{1}, \eta_{2}\right)$ are grouped in a $S_{3}$ doublet, namely $\eta$, whereas the remaining one $(\chi)$ is assigned to be a trivial $S_{3}$ singlet. The SM Higgs doublet $\phi$ is assigned to be a trivial $S_{3}$ singlet, neutral under the $Z_{8}$ discrete symmetry. Since the $S_{3}$ symmetry is preserved, the $S_{3}$ scalar doublet $\eta=\left(\eta_{1}, \eta_{2}\right)$ does not acquire a vacuum expectation value. The remaining scalar fields, i.e., $\phi$ and $\chi$, which are assigned as trivial $S_{3}$ singlets, acquire non-vanishing vacuum expectation values, as it should. Regarding the SM fermion sector, I assign the left handed fermionic fields, the right handed top quark field as trivial $S_{3}$ singlets and the remaining right handed SM fermionic fields as $S_{3}$ nontrivial singlets, implying that the top quark is the only SM charged fermion that acquires a tree level mass. The remaining SM charged fermions get their masses from a one loop radiative seesaw mechanism and the hierarchy among their masses will arise from the different $Z_{8}$ charge assignments of the fermionic fields. As it will be shown in the following, light active neutrinos masses will arise from a three loop radiative seesaw mechanism. In order that all fermions lighter than the top quark acquire non-vanishing masses, the fermion sector of the standard model is extended by including two heavy right handed Majorana neutrinos $v_{1 R}, v_{2 R}$, six SM gauge singlet charged leptons $E_{\kappa L}$ and $E_{\kappa R}(\kappa=1, \ldots, 6)$, ten heavy $S U(2)_{L}$ singlet exotic quarks $B_{\kappa L}, B_{\kappa R}(\kappa=1, \ldots, 6), T_{\lambda L}$, $T_{\lambda R}(\lambda=1, \ldots, 4)$. The heavy exotic $T_{\lambda}(\lambda=1, \ldots, 4)$ and $B_{\kappa}(\kappa=1, \ldots, 6)$ quarks should have electric charges equal to $\frac{2}{3}$ and $-\frac{1}{3}$, respectively, in order to implement a one loop radiative seesaw mechanism that generates masses for quarks, lighter than the top quark. To build the Yukawa terms invariant under the $S_{3}$ discrete group, I assign the two heavy 
right handed Majorana neutrinos as non-trivial $S_{3}$ singlets, whereas the non-SM charged fermions are grouped into the $S_{3}$ doublets $T_{L, R}^{(r)}(r=1,2), B_{L, R}^{(k)}$ and $E_{L, R}^{(k)}(k=1,2,3)$. I do not unify the two heavy right handed Majorana neutrinos in a $S_{3}$ doublet since that assignment will result in two massless active neutrinos, which is in clear contradiction with the neutrino oscillation experimental data. The non-SM fermionic fields described above, together with the $S_{3}$ doublet $\eta=\left(\eta_{1}, \eta_{2}\right)$ will induce one and three loop radiative seesaw mechanisms to generate the masses for fermions lighter than the top quark and for the light active neutrinos, respectively. The aforementioned non-SM fermion content is the minimal required to generate the masses for fermions lighter than the top quark and for the light active neutrinos. I further assume that the fermionic fields transform under the $Z_{8}$ symmetry, as follows:

$$
\begin{aligned}
q_{j L} & \rightarrow e^{-\frac{\pi i(3-j)}{2}} q_{j L}, \quad u_{j R} \rightarrow e^{\frac{\pi i(3-j)}{2}} u_{j R}, \\
d_{j R} & \rightarrow e^{\frac{\pi i(3-j)}{2}} d_{j R}, \\
l_{j L} & \rightarrow e^{-\frac{\pi i(3-j)}{2}} l_{j L}, \quad l_{j R} \rightarrow e^{\frac{\pi i(3-j)}{2}} l_{j R}, \quad j=1,2,3, \\
T_{L}^{(r)} & \rightarrow e^{-\frac{\pi i}{4}} T_{L}^{(r)}, \quad T_{R}^{(r)} \rightarrow e^{\frac{\pi i}{4}} T_{R}^{(r)}, \quad r=1,2, \\
B_{L}^{(k)} & \rightarrow e^{-\frac{\pi i}{4}} B_{L}^{(k)}, \quad B_{R}^{(k)} \rightarrow e^{\frac{\pi i}{4}} B_{R}^{(k)}, \quad k=1,2,3, \\
E_{L}^{(k)} & \rightarrow e^{-\frac{\pi i}{4}} E_{L}^{(k)}, \quad E_{R}^{(k)} \rightarrow e^{\frac{\pi i}{4}} E_{R}^{(k)}, \quad k=1,2,3, \\
v_{S R} & \rightarrow e^{-\frac{\pi i}{4}} v_{S R}, \quad s=1,2 .
\end{aligned}
$$

The EW scalar singlets $\eta=\left(\eta_{1}, \eta_{2}\right)$ and $\chi$ are charged under the $Z_{8}$ symmetry as $\eta \rightarrow e^{-\frac{\pi i}{4}} \eta, \chi \rightarrow e^{-\frac{\pi i}{2}} \chi$. With the above particle content, the following quark and charged lepton and neutrino Yukawa terms invariant under the symmetries of the model arise:

$$
\begin{aligned}
-\mathscr{L}_{\mathrm{Y}}^{(u)}= & \sum_{j=1}^{3} \sum_{r=1}^{2} y_{j r}^{(u)} \bar{q}_{j L} \widetilde{\phi}\left(T_{R}^{(r)} \eta\right)_{\mathbf{1}} \frac{\chi^{3-j}}{\Lambda^{4-j}} \\
& +\sum_{r=1}^{2} \sum_{s=1}^{2} x_{r s}^{(u)}\left(\bar{T}_{L}^{(r)} \eta\right)_{\mathbf{1}^{\prime}} u_{s R} \frac{\chi^{3-k}}{\Lambda^{3-k}} \\
& +\sum_{j=1}^{3} y_{j 3}^{(u)} \bar{q}_{j L} \widetilde{\phi}_{3 R} \frac{\chi^{3-j}}{\Lambda^{3-j}} \\
& +\sum_{r=1}^{2} y_{r}^{(T)}\left(\bar{T}_{L}^{(r)} T_{R}^{(r)}\right)_{\mathbf{1}} \chi+h \cdot c \\
\mathscr{L}_{\mathrm{Y}}^{(d)}= & \sum_{j=1}^{3} \sum_{k=1}^{3} y_{j k}^{(d)} \bar{q}_{j L} \phi\left(B_{R}^{(k)} \eta\right)_{\mathbf{1}} \frac{\chi^{3-j}}{\Lambda^{4-j}} \\
& +\sum_{j=1}^{3} \sum_{k=1}^{3} x_{j k}^{(d)}\left(\bar{B}_{L}^{(j)} \eta\right)_{\mathbf{1}^{\prime}} d_{k R} \frac{\chi^{3-k}}{\Lambda^{3-k}} \\
& +\sum_{k=1}^{3} y_{k}^{(B)}\left(\bar{B}_{L}^{(k)} B_{R}^{(k)}\right)_{\mathbf{1}} \chi+h . c .
\end{aligned}
$$

$$
\begin{aligned}
-\mathscr{L}_{\mathrm{Y}}^{(l)}= & \sum_{j=1}^{3} \sum_{k=1}^{3} y_{j k}^{(l)} \bar{l}_{j L} \phi\left(E_{R}^{(k)} \eta\right)_{\mathbf{1}} \frac{\chi^{3-j}}{\Lambda^{4-j}} \\
& +\sum_{j=1}^{3} \sum_{k=1}^{3} x_{j k}^{(l)}\left(\bar{E}_{L}^{(j)} \eta\right)_{\mathbf{1}^{\prime}} l_{k R} \frac{\chi^{3-k}}{\Lambda^{3-k}} \\
& +\sum_{k=1}^{3} y_{k}^{(E)}\left(\bar{E}_{L}^{(k)} E_{R}^{(k)}\right)_{\mathbf{1}} \chi+h . c \\
-\mathscr{L}_{\mathrm{Y}}^{(v)}= & \sum_{j=1}^{3} \sum_{s=1}^{2} y_{j s}^{(v)} \bar{l}_{j L} \widetilde{\phi}_{v_{s R}} \frac{\left[\eta^{*}\left(\eta \eta^{*}\right)_{\mathbf{2}}\right]_{\mathbf{1}^{\prime}} \chi^{3-j}}{\Lambda^{6-j}} \\
& +\sum_{s=1}^{2} y_{s} \bar{\nu}_{s R} v_{s R}^{C} \chi+h . c .
\end{aligned}
$$

Here, for the sake of simplicity, I have neglected the mixing terms between the different $S_{3}$ fermionic doublets as well as the mixings between the right handed Majorana neutrinos. I have assumed that the non-SM fermions are physical fields. After the spontaneous breaking of the electroweak and the $Z_{8}$ discrete symmetry and considering that the $S_{3}$ symmetry is preserved, the Yukawa interactions given above will generate tree level masses for the top quark and for the non-SM fermions and one loop level masses for the remaining SM charged fermions. To generate the one loop level masses for the charged fermions lighter than the top quark, the $S_{3}$ symmetry has to be softly broken by adding a $\mu_{12}^{2} \eta_{1} \eta_{2}$ term in the scalar potential for the $S_{3}$ scalar doublet $\eta=\left(\eta_{1}, \eta_{2}\right)$. Since the $S_{3}$ scalar doublet $\eta=\left(\eta_{1}, \eta_{2}\right)$ has a vanishing vacuum expectation value, light active neutrinos do not acquire tree level masses, they get masses via a three loop radiative seesaw mechanism (as follows from the $S_{3}$ invariance of the neutrino Yukawa interactions) that involves the two heavy Majorana neutrinos $v_{1 R}, v_{2}$ as well as the real and imaginary parts of the $\eta_{1}, \eta_{2}$ scalar fields running in the loops. It is remarkable that this three loop level radiative seesaw mechanism for light active neutrino masses does not require charged scalar fields as in the other three loop level mechanisms discussed in the literature [1].

Since the hierarchy of charged fermion masses and quark mixing angles arises from the breaking of the $Z_{8}$ discrete group, and in order to relate the quark masses with the quark mixing parameters, the vacuum expectation value $(\mathrm{VEV})$ of the SM scalar singlet $\chi$ is set as follows: $v_{\chi}=\lambda \Lambda$, where $\lambda=0.225$ is one of the Wolfenstein parameters and $\Lambda$ corresponds to the model cutoff.

\section{Fermion masses and mixings}

From the Yukawa terms, it follows that the quark, charged lepton, and light active neutrino mass matrices have the form 


$$
\begin{aligned}
M_{U}= & \left(\begin{array}{ccc}
\varepsilon_{11}^{(u)} \lambda^{3} & \varepsilon_{12}^{(u)} \lambda^{2} & y_{13}^{(u)} \lambda^{2} \\
\varepsilon_{21}^{(u)} \lambda^{2} & \varepsilon_{22}^{(u)} \lambda & y_{23}^{(u)} \lambda \\
\varepsilon_{31}^{(u)} \lambda & \varepsilon_{32}^{(u)} & y_{33}^{(u)}
\end{array}\right) \frac{v}{\sqrt{2}}, \\
M_{D, l}= & \left(\begin{array}{ccc}
\varepsilon_{11}^{(d, l)} \lambda^{4} & \varepsilon_{12}^{(d, l)} \lambda^{3} & \varepsilon_{13}^{(d, l)} \lambda^{2} \\
\varepsilon_{21}^{(d, l)} \lambda^{3} & \varepsilon_{22}^{(d, l)} \lambda^{2} & \varepsilon_{23}^{(d, l)} \lambda \\
\varepsilon_{31}^{(d, l)} \lambda^{2} & \varepsilon_{32}^{(d, l)} \lambda & \varepsilon_{33}^{(d, l)}
\end{array}\right) \frac{v}{\sqrt{2}}, \\
M_{v}= & \left(\begin{array}{ccc}
W_{1}^{2} & W_{1} W_{2} \cos \varphi & W_{1} W_{3} \cos (\varphi-\rho) \\
W_{1} W_{2} \cos \varphi & W_{2}^{2} & W_{2} W_{3} \cos \rho \\
W_{1} W_{3} \cos (\varphi-\rho) & W_{2} W_{3} \cos \rho & W_{3}^{2}
\end{array}\right),
\end{aligned}
$$

where

$$
\begin{aligned}
\vec{W}_{j} & =\left(\frac{A_{j 1} \sqrt{y_{1} v_{\chi} f_{1}^{(v)}}}{64 \pi^{3} \Lambda}, \frac{A_{j 2} \sqrt{y_{2} v_{\chi} f_{2}^{(v)}}}{64 \pi^{3} \Lambda}\right), \quad j=1,2,3 \\
\cos \varphi & =\frac{\vec{W}_{1} \cdot \vec{W}_{2}}{\left|\vec{W}_{1}\right|\left|\vec{W}_{2}\right|}, \quad \cos (\varphi-\rho)=\frac{\vec{W}_{1} \cdot \vec{W}_{3}}{\left|\vec{W}_{1}\right|\left|\vec{W}_{3}\right|}, \quad W_{j}=\left|\vec{W}_{j}\right|, \\
\cos \rho & =\frac{\vec{W}_{2} \cdot \vec{W}_{3}}{\left|\vec{W}_{2}\right|\left|\vec{W}_{3}\right|}, \quad A_{j s}=\lambda^{3-j} y_{j s}^{(v)} \frac{v}{\sqrt{2}}, \quad s=1,2,
\end{aligned}
$$

where $\varepsilon_{j k}^{(f)}(j, k=1,2,3)$ with $f=u, d, l$ and $f_{s}^{(v)}$ $(s=1,2)$ are dimensionless parameters generated at one and three loop levels, respectively. Furthermore, the entries of the charged lepton and down type quark mass matrices exhibit the same scalings in terms of the Wolfenstein parameter $\lambda$, as seen from Eq. (7). Since the dimensionless parameters $\varepsilon_{j k}^{(f)}$ $(j, k=1,2,3)$ with $f=u, d, l$, are generated at one loop level, I set $\varepsilon_{j k}^{(f)}=a_{j k}^{(f)} \lambda^{3}$, where $a_{j k}^{(f)}$ are $\mathscr{O}(1)$ parameters. Since the charged fermion mass and quark mixing angles is caused by the breaking of the $Z_{8}$ discrete group and in order to simplify the analysis, I adopt a benchmark where I set

$a_{12}^{(u)}=a_{21}^{(u)}, \quad a_{31}^{(u)}=y_{13}^{(u)}, \quad a_{32}^{(u)}=y_{23}^{(u)}$

$a_{12}^{(d)}=\left|a_{12}^{(d)}\right| e^{-i \tau_{1}}, \quad a_{21}^{(d)}=\left|a_{12}^{(d)}\right| e^{i \tau_{1}}$,

$a_{13}^{(d)}=\left|a_{13}^{(d)}\right| e^{-i \tau_{2}}, \quad a_{31}^{(d)}=\left|a_{13}^{(d)}\right| e^{i \tau_{2}}, \quad a_{23}^{(d)}=a_{32}^{(d)}$.

Furthermore I set $a_{33}^{(u)}=1$, as suggested by naturalness arguments. Then I proceed to fit the effective parameters $a_{11}^{(u)}, a_{22}^{(u)}, a_{12}^{(u)}, a_{13}^{(u)}, a_{23}^{(u)}, a_{11}^{(d)}, a_{22}^{(d)}, a_{33}^{(d)},\left|a_{12}^{(d)}\right|$, $\left|a_{13}^{(d)}\right|, a_{23}^{(d)}$ and the phases $\tau_{1}, \tau_{2}$, to reproduce the experimental values of the quark masses, the three quark mixing angles and the CP violating phase $\delta$. The values they obtained, found in Table 1, correspond to the best-fit values:

$a_{11}^{(u)} \simeq 0.58, \quad a_{22}^{(u)} \simeq 2.19, \quad a_{12}^{(u)} \simeq 0.67$,

$a_{13}^{(u)} \simeq 0.80, \quad a_{23}^{(u)} \simeq 0.83, \quad a_{11}^{(d)} \simeq 1.96$,
Table 1 Model and experimental values of the quark masses and CKM parameters

\begin{tabular}{lll}
\hline Observable & Model value & Experimental value \\
\hline$m_{u}(\mathrm{MeV})$ & 1.44 & $1.45_{-0.45}^{+0.56}$ \\
$m_{c}(\mathrm{MeV})$ & 656 & $635 \pm 86$ \\
$m_{t}(\mathrm{GeV})$ & 177.1 & $172.1 \pm 0.6 \pm 0.9$ \\
$m_{d}(\mathrm{MeV})$ & 2.9 & $2.9_{-0.4}^{+0.5}$ \\
$m_{s}(\mathrm{MeV})$ & 57.7 & $57.7_{-15.7}^{+16.8}$ \\
$m_{b}(\mathrm{GeV})$ & 2.82 & $2.82_{-0.04}^{+0.09}$ \\
$\sin \theta_{12}$ & 0.225 & 0.225 \\
$\sin \theta_{23}$ & 0.0412 & 0.0412 \\
$\sin \theta_{13}$ & 0.00351 & 0.00351 \\
$\delta$ & $64^{\circ}$ & $68^{\circ}$ \\
\hline
\end{tabular}

$a_{12}^{(d)} \simeq 0.53, \quad a_{13}^{(d)} \simeq 1.07, \quad a_{22}^{(d)} \simeq 1.93$,

$a_{23}^{(d)} \simeq 1.36, \quad a_{33}^{(d)} \simeq 1.35, \quad \tau_{1} \simeq 9.56^{\circ}, \quad \tau_{2} \simeq 4.64^{\circ}$.

The obtained quark masses, quark mixing angles and CP violating phase are consistent with the experimental data. The obtained and experimental values for the physical observables of the quark sector are reported in Table 1. I use the experimental values of the quark masses at the $M_{Z}$ scale, from Ref. [2], whereas the experimental values of the CKM parameters are taken from Ref. [3].

In the following I will explain the reason for choosing the $Z_{8}$ discrete symmetry. It is noteworthy that the $Z_{4}$ discrete group is the smallest cyclic symmetry that allows one to get the $\lambda^{2}$ suppression in the 13 entry of the up type quark mass matrix from a $\frac{\chi^{2}}{\Lambda^{2}}$ insertion on the $\bar{q}_{1 L} \widetilde{\phi} u_{3 R}$ operator. However, since the masses of the non-SM fermions arise from their renormalizable Yukawa interactions with the SM scalar singlet $\chi$, charged under the discrete cyclic group, the invariance of these Yukawa interactions under the cyclic symmetry requires one to consider the $Z_{8}$ instead of the $Z_{4}$ discrete symmetry.

In the concerning to the charged lepton sector, I adopt a benchmark where I set $a_{j k}^{(l)}=a_{k}^{(l)} \delta_{j k}$. This benchmark scenario that leads to a diagonal charged lepton mass matrix is justified by the requirement of forbidding unobserved lepton flavor violating processes such as $\mu \rightarrow e \gamma$. This assumption is also made in the framework of an extended inert two Higgs doublet model addressed to explain the charged lepton mass hierarchy [4]. Within the benchmark previously described, the charged lepton masses take the form:

$m_{e}=a_{1}^{(l)} \lambda^{7} \frac{v}{\sqrt{2}}, \quad m_{\mu}=a_{2}^{(l)} \lambda^{5} \frac{v}{\sqrt{2}}, \quad m_{\tau}=a_{3}^{(l)} \lambda^{3} \frac{v}{\sqrt{2}}$. 
Regarding the neutrino sector, from the light active neutrino mass texture $M_{\nu}$, it follows that the light active neutrino spectrum includes one massless neutrino and two massive neutrinos for both normal and inverted neutrino mass hierarchies. It is noteworthy that two heavy Majorana neutrinos are needed in this model to generate the two non-vanishing neutrino mass squared splittings measured in neutrino oscillation experiments. Furthermore, due to the preserved $S_{3}$ symmetry, the model allows for stable dark matter candidates, which could be either the right handed Majorana neutrinos $v_{S R}$ or the lightest of the scalar fields $\operatorname{Re}\left(\eta_{s}\right)$ and $\operatorname{Im}\left(\eta_{S}\right)$ $(s=1,2)$. In order to show that the light active neutrino texture $M_{v}$ can fit the experimental data, I set $\varphi=2 \rho$ only for the case of normal hierarchy $(\mathrm{NH})$. Varying the lepton sector model parameters $a_{1}^{(l)}, a_{2}^{(l)}, a_{3}^{(l)}, \rho, W_{1}, W_{2}$, and $W_{3}$ (as well as $\varphi$ for IH only), I fitted the charged lepton masses, the neutrino mass squared splittings $\Delta m_{21}^{2}, \Delta m_{31}^{2}$ and the leptonic mixing parameters $\sin ^{2} \theta_{12}, \sin ^{2} \theta_{13}$, and $\sin ^{2} \theta_{23}$ to their experimental values for $\mathrm{NH}$ and $\mathrm{IH}$. The results shown in Table 2 correspond to the following best-fit values:

$$
\begin{aligned}
& \rho \simeq 38.73^{\circ}, \quad W_{1} \simeq-0.063 \mathrm{eV}^{\frac{1}{2}}, \quad W_{2} \simeq 0.18 \mathrm{eV}^{\frac{1}{2}}, \\
& W_{3} \simeq 0.15 \mathrm{eV}^{\frac{1}{2}}, \quad \text { for } \mathrm{NH} \\
& a_{1}^{(l)} \simeq 0.1, \quad a_{2}^{(l)} \simeq 1.02, \quad a_{3}^{(l)} \simeq 0.88 \\
& \rho \simeq 162.26^{\circ}, \quad \varphi \simeq 79.44^{\circ}, \quad W_{1} \simeq 0.22 \mathrm{eV}^{\frac{1}{2}} \\
& W_{2} \simeq 0.15 \mathrm{eV}^{\frac{1}{2}}, \quad W_{3} \simeq 0.17 \mathrm{eV}^{\frac{1}{2}}, \quad \text { for IH } \\
& a_{1}^{(l)} \simeq 0.1, \quad a_{2}^{(l)} \simeq 1.02, \quad a_{3}^{(l)} \simeq 0.88,
\end{aligned}
$$

Using the best-fit values given above, I get for $\mathrm{NH}$ and $\mathrm{IH}$, respectively, the following neutrino masses:

$m_{1}=0, \quad m_{2} \approx 9 \mathrm{meV}, \quad m_{3} \approx 50 \mathrm{meV}, \quad$ for $\mathrm{NH}$

$m_{1} \approx 49 \mathrm{meV}, \quad m_{2} \approx 50 \mathrm{meV}, \quad m_{3}=0$, for IH

The obtained and experimental values of the observables in the lepton sector are shown in Table 2. The experimental values of the charged lepton masses, which are given at the $M_{Z}$ scale, have been taken from Ref. [2], whereas the experimental values of the neutrino mass squared splittings and leptonic mixing angles for both normal (NH) and inverted (IH) mass hierarchies, are taken from Ref. [5]. The obtained charged lepton masses, neutrino mass squared splittings and lepton mixing angles are in excellent agreement with the experimental data for both normal and inverted neutrino mass hierarchies. For the sake of simplicity, I assumed all leptonic parameters to be real, but a non-vanishing $\mathrm{CP}$ violating phase in the PMNS mixing matrix can be generated by making one of the entries of the neutrino mass matrix $M_{v}$ to be complex. It is noteworthy to mention that the consistency of the Higgs couplings to SM fermions and
Table 2 Model and experimental values of the charged lepton masses, neutrino mass squared splittings, and leptonic mixing parameters for the normal $(\mathrm{NH})$ and inverted $(\mathrm{IH})$ mass hierarchies

\begin{tabular}{lll}
\hline Observable & Model value & Experimental value \\
\hline$m_{e}(\mathrm{MeV})$ & 0.487 & 0.487 \\
$m_{\mu}(\mathrm{MeV})$ & 102.8 & $102.8 \pm 0.0003$ \\
$m_{\tau}(\mathrm{GeV})$ & 1.75 & $1.75 \pm 0.0003$ \\
$\Delta m_{21}^{2}\left(10^{-5} \mathrm{eV}^{2}\right)(\mathrm{NH})$ & 7.22 & $7.60_{-0.18}^{+0.19}$ \\
$\Delta m_{31}^{2}\left(10^{-3} \mathrm{eV}^{2}\right)(\mathrm{NH})$ & 2.50 & $2.48_{-0.07}^{+0.05}$ \\
$\sin ^{2} \theta_{12}(\mathrm{NH})$ & 0.334 & $0.323 \pm 0.016$ \\
$\sin ^{2} \theta_{23}(\mathrm{NH})$ & 0.567 & $0.567_{-0.128}^{+0.032}$ \\
$\sin ^{2} \theta_{13}(\mathrm{NH})$ & 0.0228 & $0.0234 \pm 0.0020$ \\
$\Delta m_{21}^{2}\left(10^{-5} \mathrm{eV}^{2}\right)(\mathrm{IH})$ & 7.60 & $7.60_{-0.18}^{+0.19}$ \\
$\Delta m_{13}^{2}\left(10^{-3} \mathrm{eV}^{2}\right)(\mathrm{IH})$ & 2.48 & $2.48_{-0.06}^{+0.05}$ \\
$\sin ^{2} \theta_{12}(\mathrm{IH})$ & 0.323 & $0.323 \pm 0.016$ \\
$\sin ^{2} \theta_{23}(\mathrm{IH})$ & 0.573 & $0.573_{-0.043}^{+0.025}$ \\
$\sin ^{2} \theta_{13}(\mathrm{IH})$ & 0.0240 & $0.0240 \pm 0.0019$ \\
\hline
\end{tabular}

gauge bosons with the SM expectation requires that the mixing between the $126 \mathrm{GeV}$ Higgs and the SM scalar singlet $\chi$ to be suppressed. In addition, the model cutoff has to be of the order of few TeVs to prevent the one loop level masses and Yukawa couplings for charged fermions lighter than the top quark to be arbitrary small. An ultraviolet completion of this model will consist in replacing the EW scalar singlets $\eta=\left(\eta_{1}, \eta_{2}\right)$ by two $S U$ (2) inert scalar doublets unified in a $S_{3}$ doublet. This will make the model cutoff very high thus avoiding large effects in the low energy flavor physics (FCNC etc.).

\section{Conclusions}

I have proposed the first multiscalar singlet extension of the SM that generates one and three loop level masses for charged fermions lighter than the top quark and for the light active neutrinos, respectively, via radiative seesaw mechanisms, without invoking electrically charged scalar fields. These one and three loop radiative seesaw mechanisms are mediated by the $\eta_{1}$ and $\eta_{2}$ scalar fields as well as by exotic charged fermions and right handed Majorana neutrinos, respectively. The model has the $S_{3} \times Z_{8}$ discrete symmetry, where $S_{3}$ is preserved and $Z_{8}$ is spontaneously broken. The breaking of the $Z_{8}$ discrete group generates the non-SM fermion masses as well as the observed SM charged fermion mass and quark mixing pattern. The unbroken $S_{3}$ symmetry of the model allows for natural dark matter candidates, which could be either the right handed Majorana neutrinos $v_{s}(s=1,2)$ or the lightest of the $S_{3}$ charged scalar fields $\operatorname{Re}\left(\eta_{1}\right), \operatorname{Re}\left(\eta_{2}\right)$, 
$\operatorname{Im}\left(\eta_{1}\right)$, and $\operatorname{Im}\left(\eta_{2}\right)$. This possibility is beyond the scope of this article.

Acknowledgments I am very grateful to Professor Riccardo Barbieri for the careful reading of this article.

Open Access This article is distributed under the terms of the Creative Commons Attribution 4.0 International License (http://creativecomm ons.org/licenses/by/4.0/), which permits unrestricted use, distribution, and reproduction in any medium, provided you give appropriate credit to the original author(s) and the source, provide a link to the Creative Commons license, and indicate if changes were made.

Funded by SCOAP ${ }^{3}$.

\section{References}

1. E. Ma, Phys. Rev. Lett. 115(1), 011801 (2015)

2. K. Bora, Horizon 2 (2013). arXiv:1206.5909 [hep-ph]

3. K.A. Olive et al., Chin. Phys. C 38, 090001 (2014)

4. H. Okada, K. Yagyu, Phys. Rev. D 89(5), 053008 (2014)

5. D.V. Forero, M. Tortola, J.W.F. Valle, Phys. Rev. D 90(9), 093006 (2014) 